\title{
Measuring Thresholds of Serum Lipid Level related to Hypertension and Age Using Receiver Operating Characteristic Curve in Korean Adult
}

\author{
Seong-gil Kim, PT, PhD, So-Hyun Park, PT, $\mathrm{PhD}^{\dagger}$
}

Department of Physical Therapy, Youngsan University

Received: June 30, 2014 / Revised: July 9, 2014 / Accepted: August 20, 2014

(C) 2014 J Korean Soc Phys Med

\section{| Abstract |}

PURPOSE: The purpose of this study was to determine the optimal thresholds of serum lipid level related to hypertension according to age in Korean adults.

METHODS: In total, 564 adults who visited the health examine center in general hospital were included. The blood pressure and lipid profiles of low density lipoprotein cholesterol(LDL), high density lipoprotein cholesterol (HDL), triglyceride(TG) were measured. Receiver operating characteristic (ROC) curve were used to establish optimal thresholds between blood pressure and lipid profiles.

RESULTS: The optimal TG cutoff value were $110.50(\mathrm{mg} / \mathrm{dl})$ in the $18-39$ and $81.50(\mathrm{mg} / \mathrm{dl})$ in the $40-59$ age group, and optimal cutoff value of LDL were $126.50(\mathrm{mg} / \mathrm{dl})$ in the 40-59 age group and $111.00(\mathrm{mg} / \mathrm{dl})$ in the 60 and over age group. There was a negative correlation between HDL and hypertension, a higher HDL decreased hypertension. The optimal cutoff value of HDL was $49.50(\mathrm{mg} / \mathrm{dl})$ in the $18-39$ age group.

CONCLUSION: The thresholds of hypertension were

†Corresponding Author: ptpsh@ysu.ac.kr

This is an Open Access article distributed under the terms of the Creative Commons Attribution Non-Commercial License (http://creativecommons.org/licenses/by-nc/3.0) which permits unrestricted non-commercial use, distribution, and reproduction in any medium, provided the original work is properly cited. lower in LDL and TG with aging. This result indicated that elderly people needs to concern more about their lipid profiles to maintain healthy cardiovascular function.

Key Words: Aging, Blood Pressure, Lipid

\section{Introduction}

In south Korea, stroke and coronary heart disease are the major cause of death(Statistics Korea, 2012) and the cardiovascular disease increase as we becoming an aging society(Stern et al. 2003). Recently, cardiovascular physical therapy has become the fastest growing area; however, research in this field is still deficient in Korea. As the fundamental measurements of health examination, blood pressures and serum lipid level such as low-density lipoprotein cholesterol(LDL), high-density lipoprotein cholesterol(HDL), and triglyceride(TG) are commonly used to determine cardiovascular health(Mann et al, 2011). Therefore understanding these levels is essential for physiotherapist.

In particular, hypertension is strongly, positively correlated with cardiovascular disease(Mann et al, 2011). Statistically, one out of three adults over the age of 30 in Korea has hypertension(Statistics Korea, 2012). Therefore 
many researchers tried to manage hypertension and studied its risk factors. It is well known that ageing, obesity, and hyperlipidaemia are closely related(Stern et al. 2003). However, diverse studies suggested. For example, $\operatorname{Kim}(2011)$ reported that the increasing factors of blood pressure are TC, TG, and age, but Eom et al(2008) reported that mean serum cholesterol did not affected on hypertension. Furthermore, Jung et al(2007) have reported that $\mathrm{TG}$ was lower in the normal group than the hypertension obesity group, but total cholesterol, HDL and LDL are not related to hypertension. We hypothesize that these results were derived from age factors.

According to National Cholesterol Education Program (2014), the optimal range of TC score, calculated by HDL plus LDL plus $20 \%$ of TG level is less than $200 \mathrm{mg} / \mathrm{dL}$. Having a LDL and TG of less than 100 and $150 \mathrm{mg} / \mathrm{dL}$ are ideal for normal people. A HDL level of $60 \mathrm{mg} / \mathrm{dL}$ or higher helps protect against heart disease. As well as these normal ranges, it is worth to research the thresholds of each serum lipid level that trigger hypertension to learn whether there is a relationship between lipid profiles and hypertension by age. However, to our knowledge, there is little evidence about the thresholds of serum lipid level related to the hypertension.

Therefore, the purpose of this study is to analyze the relationship between hypertension and lipid profiles by age and determine the optimal thresholds of serum lipid level related to hypertension and age in Korean adults.

\section{SUBJECTS AND METHODS}

\section{Subjects}

This study was conducted as a cross-sectional measurement study. Subjects who visited the health examine center in general hospital were included from Apr. 1, 2010 to Aug. 15 , 2013. Subjects with a history or present case (as determined by the interviews or laboratory tests) of any diseases of the parathyroid gland, and diabetes mellitus, and those who had ever taken drugs that affect hypertension, hyperlipidaemia and metabolic syndrome are excluded. The 564 subjects were included in this study. Included subjects were sorted according to their age as follows; young (ages 18-39), middle(ages 40-59), old(ages 60 and older) group.

The principal objective and all study procedures of this study were explained to subjects and written informed consent was taken before the study. This protocol was conducted in accordance with the ethical standards of the Declaration of Helsinki.

\section{Procedures}

All subjects underwent a full physical examination, blood investigations, and morphometric evaluation.

The blood pressure was measured using an automatic blood pressure monitor(BP-203 RV II, colin corp., Aichi, Japan). Hypertension is defined as systolic and diastolic blood pressure above $140 \mathrm{mmHg}$ and $90 \mathrm{mmHg}$ respectably (National Institute of Health, 2003).

Nonfasting blood samples were obtained. All the serological testing were referred to the hospital associated companies and they were measured by routine laboratory method. The LDL and HDL were measured using enzymatic colorimetric method with Hitach 7600 chemical analyzer(Hitachi co., Ltd, Tokyo, Japan) and TG was measured using lipase, GK, GPO, POD with glycerol blank.

\section{Statistics}

General characteristic was expressed via descriptive analysis. Receiver operating characteristic (ROC) curve was used to establish optimal thresholds between blood pressure and lipid profiles.

Cutoff values, sensitivity, and specificity about scale can be analyzed through ROC curves and it is the widely used statistical methods not only in public health but in medical field. For example, cutoff value for a disease indicates a division point for the existence of disease, 
sensitivity indicates the possibility of the clinical examination to identify a patient with his disease, and specificity indicates the possibility of the clinical examination to identify a patient without his disease. The whole area under the curve(AUC) is 1 and this area has significant meaning when it is lower than 0.5 and the bigger number makes the test more accurate(Zweig \& Campbell, 1993).

SPSS 21.0 for Windows was used, and statistical significance was accepted for $p$-values of $<0.05$.

\section{Results}

1. General characteristics of subjects

Table 1. General characteristics of subjects

$(n=564)$

\begin{tabular}{cccc}
\hline SEX(M/F) & Age & Total(N) & $\begin{array}{c}\text { Hypertension/ } \\
\text { normal }\end{array}$ \\
\hline $64 / 56$ & $18-39$ & 120 & $41 / 79$ \\
$229 / 146$ & $40-59$ & 375 & $129 / 246$ \\
$38 / 31$ & $\geqq 60$ & 69 & $28 / 41$ \\
\hline
\end{tabular}

Tablel shows the general characteristics of subjects. Total subject was 564 divided into three groups which were 18-39, 40-59, and 60 and over.

\section{AUC and cut-off value of Serum lipid for hypertension}

The optimal cut-off value of serum lipid level related to hypertension and age in the subjects and years surveyed is estimated by using ROC curve (Table 2).

There were significant effects in the age ranges 18-39 and 40-59 in TG $(\mathrm{p}<0.05)$, and the AUG in the 18-39 age group was 0.685 and 0.643 for the $40-59$ age group. There were significant effects in the 40-59 and 60 and above age groups in LDL $(p<0.05)$, and AUC in the 40-59 age group was 0.656 and 60 and over 0.650 . There was a significant effect only in the age group of 18-39 in HDL $(\mathrm{p}<0.05)$, and its AUC was 0.628.

The optimal cut-off value of TG were $110.50(\mathrm{mg} / \mathrm{dl})$ in the 18-39 and $81.50(\mathrm{mg} / \mathrm{dl})$ in the $40-59$, and optimal cut-off value of LDL were $126.50(\mathrm{mg} / \mathrm{dl})$ in the 40-59 and $111.00(\mathrm{mg} / \mathrm{dl})$ in the 60 and over. There was negative correlation between HDL and hypertension, and higher HDL decreased hypertension. The optimal cut-off value of HDL was $49.50(\mathrm{mg} / \mathrm{dl})$ in the 18-39 age group.

Table 2. Serum lipid level for hypertension using ROC curve

\begin{tabular}{cccccc}
\hline Lipid & Age & Cut-off & SS & SP & AUC(95\%CI) \\
\hline TG & $18-39$ & 110.50 & .634 & .734 & $.685(.585-.785)^{*}$ \\
& $40-59$ & 81.50 & .729 & .504 & $.643(.584-.701)^{*}$ \\
& $\geqq 60$ & 137.50 & .464 & .829 & $.591(.451-.731)$ \\
\hline LDL & $18-39$ & 126.00 & .634 & .443 & $.526(.415-.636)$ \\
& $40-59$ & 126.50 & .674 & .602 & $.656(.599-.714)^{*}$ \\
& $\geqq 60$ & 111.00 & .821 & .512 & $.650(.519-.781)^{*}$ \\
\hline HDL & $18-39$ & 49.50 & .646 & .561 & $.628(.527-.729)^{*}$ \\
& $40-59$ & 54.50 & .516 & .651 & $.554(.493-.615)$ \\
& $\geqq 60$ & 57.50 & .366 & .679 & $.467(.327-.607)$ \\
\hline
\end{tabular}

${ }^{*} \mathrm{p}<0.05$, AUC: area under the curve SS: sensitivity, SP: specificity, TG: triglyceride, LDL: low density lipoprotein, HDL: high density lipoprotein 


\section{Discussion}

Hypertension is one of the main potential risk factors for many diseases and it may develop into life-threatening diseases such as coronary heart disease(CHD)(Martin et al, 1986). Generally, serum lipid level is one of the main factors that affects hypertension, and a higher serum lipid level increases the blood pressure causing hypertension. In particular, HDL, LDL, and TG are known to affect hypertension among these factors (Sander \& Giles, 2002). However, the effects of factors on serum lipid level, hypertension, and standards vary according to age; therefore, AUC, the thresholds of hypertension based on HDL, LDL, TG, and the optimal cut-off value were examined using ROC curve analysis in this study.

TG had significant thresholds in the younger group (18-39), and the middle aged group (40-59) and older aged groups had relatively lower optimal cut-off values. The younger group had a $110.50(\mathrm{mg} / \mathrm{dl})$ threshold and the middle aged group had an $81.50(\mathrm{mg} / \mathrm{dl})$ threshold for TG in this study. It is well known that a higher TG level increases hypertension, but this was not applied to the elderly subjects in this study, which analyzed cut-off values according to age (Miller et al, 2011). This study had lower cut-off values than commonly-known TG values. There was a relatively significant result only for the younger group, which is considered a consequence of racial differences; black people have a lower TG level than white people (Lin et al, 2011).

HDL had cut-off value of $49.50(\mathrm{mg} / \mathrm{dl})$ in the younger group (18-39) and the lower values were classified as hypertension. There were no significant results in the middle aged (40-59) or elderly group (60 and over). Higher HDL and lower TG are known to decrease hypertension, but in this study, HDL was less effective than other serum lipid factors in the middle aged and elderly groups, since it only had a significant result in the younger group (18-39)(Couillard et al, 2001).
LDL was not significant in the younger group(18-39), LDL had a $126.50(\mathrm{mg} / \mathrm{dl})$ threshold in the middle aged group (40-59), and a $111.00(\mathrm{mg} / \mathrm{dl})$ threshold in the elderly group(60 and over). This means that the older group had a lower cut-off value. The younger group was not particularly affected by LDL and in the results, older people had a lower cut off value, which shows that people get more sensitive to and are more affected by LDL as they get older. The previous study showed that the risk of hypertension increases as people get old because of their increased serum lipid level, and hypertension is affected by every serum lipid factor, particularly by an increased LDL, whereas an increased TG or decreased HDL are relatively less effective(Collaboration et al, 2007; Sander $\&$ Giles, 2002). This is consistent with the results of this study, which found that LDL is relatively more effective than HDL, which only had a significant result in the younger group, or TG, which had no significant result in the elderly group, and people were more affected by an increased LDL as they get older. Because the cut off value for LDL decreased as people got older, and people were more affected by LDL, the elderly should keep in good condition and focus more on their LDL values. Hypertension is the potential risk factor for every disease and very important not only in physical therapy but also heath care field. Hypertension can be managed focusing on the maintaining optimal value of serum lipid factors according to age through out physical therapy intervention such as various aerobic exercise.

The limitations of this research are that it only analyzed serum lipid factors related to hypertension, but could not investigate other factors such as smoking or obesity, which should be discussed in further studies.

\section{Conclusion}

This study was analyzed the optimal cut-off values of 
serum lipid levels related to hypertension and age in Korean adults and the results showed the thresholds of hypertension were lower in LDL and TG with aging. This results indicated that elderly people needs to concern more about their lipid profiles to maintain healthy cardiovascular function.

\section{References}

Collaboration PS, Lewington S, Whitlock G et al. Blood cholesterol and vascular mortality by age, sex, and blood pressure: a meta-analysis of individual data from 61 prospective studies with 55,000 vascular deaths. Lancet. 2007;370(1829):1839.

Couillard C, Després J-P, Lamarche B et al. Effects of endurance exercise training on plasma HDL cholesterol levels depend on levels of triglycerides evidence from men of the Health, Risk Factors, Exercise Training and Genetics (HERITAGE) Family Study. Arteriosclerosis, thrombosis, and vascular biology. 2001;21(7):122632.

Eom JS, Lee TR, Park SJ et al. The risk factors of the prehypertension and hypertension of rural inhabitants in chungnam-do. Korean J Nutr. 2008;41(8):742-753.

Jung JL, Lee JH, Kang SJ. The comparison to the health fitness, blood glucose and serum lipids profiles according to obesity level in hypertension patients. Journal of Korea sport research. 2007;18(4):33-40.

Kim CG. Effect of Health Behavior and Obesity Indices on Blood Pressure in 20s Man. Journal of Korean Contens.2011;11(8):231-8.
Lin SX, Carnethon M, Szklo M et al. Racial/ethnic differences in the association of triglycerides with other metabolic syndrome components: the Multi-Ethnic Study of Atherosclerosis. Metabolic syndrome and related disorders. 2011;9(1):35-40.

Mann DL, Zipes DP, Libby P et al. Braunwald's Heart Disease: A Textbook of Cardiovascular Medicine. 9th ed. Philadelphia, Pa: Saunders Elsevier; 2011:chap 47.

Martin M, Browner W, Hulley S et al. Serum cholesterol, blood pressure, and mortality: implications from a cohort of 361662 men. The Lancet. 1986;328(8513): 933-36.

Miller M, Stone NJ, Ballantyne C et al. Triglycerides and cardiovascular disease a scientific statement from the American Heart Association. Circulation. 2011;123 (20):2292-333.

National Cholesterol Education Program. ATP III Guidelines At-A-Glance Quick Desk Reference. 2014. https:// www.nhlbi.nih.gov/guidelines/cholesterol/atglance. pdf

National Institute of Health. The seventh report of the Joint National Committee on prevention, evaluation and treatment of high blood pressure. 2003.

Sander GEGiles TD. Hypertension and lipids: lipid factors in the hypertension syndrome. Current hypertension reports. 2002;4(6):458-63.

Statistics Korea. Cause of death. 2012. http://kostat.go.kr

Stern S, Behar S, Gottlieb S. Cardiology patient pages. Aging and diseases of the heart. Circulation 108(14):e99-101.

Zweig, M. H., \& Campbell, G. Receiver-operating characteristic (ROC) plots: a fundamental evaluation tool in clinical medicine. Clinical chemistry. 1993;39(4):561-77. 